\title{
Evidence for beneficial effects of compromised gastric inhibitory polypeptide action in obesity-related diabetes and possible therapeutic implications
}

\author{
N. Irwin • P. R. Flatt
}

Received: 23 March 2009 /Accepted: 25 May 2009/Published online: 17 June 2009

(C) Springer-Verlag 2009

\begin{abstract}
Gastric inhibitory polypeptide (GIP) is a physiological gut peptide secreted from the intestinal K-cells with well documented insulin-releasing actions. However, the GIP receptor is widely distributed in peripheral organs, including the pancreas, gut, adipose tissue, heart, adrenal cortex and brain, suggesting that it may have other functions. The presence of functional GIP receptors on adipocytes and the key role played by GIP in lipid metabolism and fat deposition suggest a possible beneficial effect of compromised GIP action in obesity and insulin resistance. Several key observations in animal models of obesity-related diabetes with chemically or genetically mediated biological GIP deficiency support this concept. Thus, obese diabetic animals with compromised GIP action due to peptide-based GIP receptor antagonists, small molecular weight GIP receptor antagonists, vaccination against GIP, genetic knockout of GIP receptor or targeted K-cell destruction are protected against obesity and associated metabolic disturbances. In addition, by causing preferential oxidation of fat, blockade of GIP signalling clears triacylglycerol deposits from liver and muscle, thereby restoring mechanisms for suppression of hepatic glucose output and improving insulin sensitivity. Emerging evidence also suggests that rapid cure of diabetes in grossly obese patients undergoing bypass surgery is mediated, in part, by surgical removal of GIP-secreting K-cells in the upper small intestine.
\end{abstract}

Keywords Bypass surgery - Gastric inhibitory polypeptide (GIP) · GIP receptor $\cdot$ Insulin resistance $\cdot \mathrm{K}$-cell .

Obesity $\cdot$ Review

N. Irwin $(\bowtie) \cdot$ P. R. Flatt

School of Biomedical Sciences, University of Ulster,

Cromore Road,

Coleraine, Co. Londonderry BT52 1SA, UK

e-mail: n.irwin@ulster.ac.uk

\author{
Abbreviations \\ GIP Gastric inhibitory polypeptide \\ GLP-1 Glucagon-like peptide-1 \\ GPCR G-protein coupled receptor \\ Pro $^{3} \quad$ Proline $^{3}$
}

\section{Introduction}

Gastric inhibitory polypeptide (GIP) is a 42 amino acid peptide hormone released from the proximal small intestine (duodenum and jejunum) in response to feeding [1] (Fig. 1). GIP is synthesised in intestinal K-cells and its secretion is regulated largely by the ingestion of carbohydrate and fat [2]. As such, GIP is widely recognised as a physiological incretin hormone, which potently stimulates insulin release in a glucose-dependent manner [3]. More recently, GIP has been shown to exert other effects on the pancreatic beta cell, including stimulation of proinsulin gene transcription and translation plus enhancement of beta cell growth, differentiation, proliferation and survival [4]. However, the demonstrated presence of GIP receptors outside the pancreas and stomach, and specifically on adipocytes [5], has prompted renewed awareness of GIPmediated effects on lipid metabolism and fat deposition [6]. There is substantial, growing evidence to suggest that GIP receptor-mediated effects are a key link between consumption of energy-rich high-fat diets and the development of obesity, insulin resistance and type 2 diabetes (Fig. 1; [6]). Thus, inhibition of GIP signalling could be a potential avenue for the treatment of obesity and associated complications. Accordingly, several preclinical studies have now revealed beneficial metabolic effects of sustained functional deficiency of GIP [see below]. This short review focuses on 


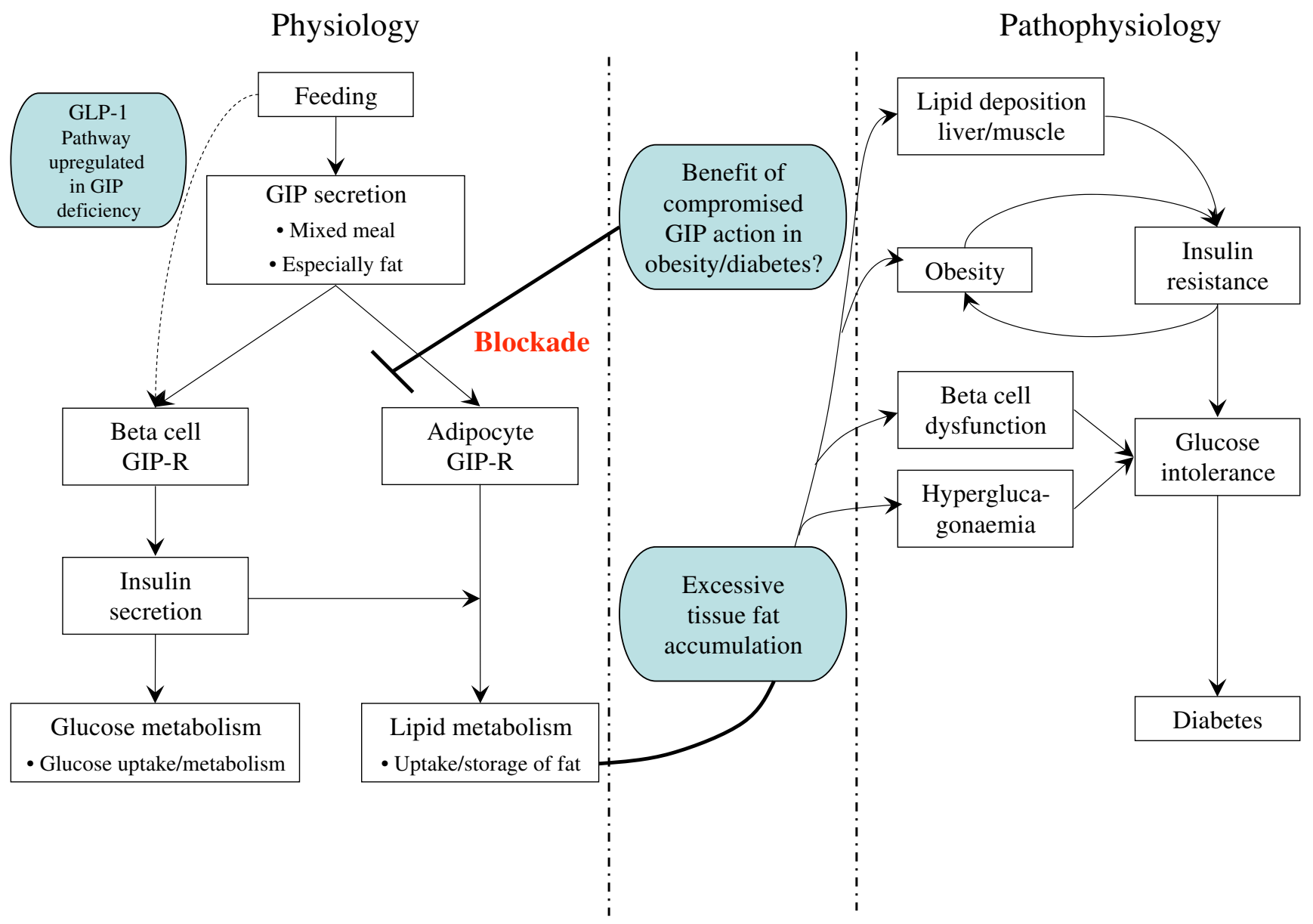

Fig. 1 Simplified scheme showing physiological roles of GIP in glucose and lipid metabolism, together with possible involvement in pathophysiology and treatment of obesity, insulin resistance and diabetes. Key physiological roles of GIP secretion in response to feeding include stimulation of insulin secretion (together with the other incretin hormone GLP-1) and promotion of fat storage mediated through adipocyte GIP receptors (GIP-R). In pathophysiology, consumption of energy-rich high-fat diet (powerful stimulus for GIP), together with positive energy balance, leads to obesity and harmful lipid deposition in liver, muscle and islet cells, giving rise to mounting insulin resistance and major disturbances in the secretion of insulin and glucagon. This scenario promotes glucose intolerance and

aspects of experimentally induced deficiency of GIP receptor-mediated actions and evidence of beneficial effects in obese insulin-resistant states. The potential of emerging therapeutic avenues and apparent parallels with bypass surgery for treatment of gross obesity are also discussed.

\section{GIP receptor antagonism with $\left(\right.$ Proline $\left.^{3}\right)$ GIP}

(Proline $^{3}\left[\right.$ Pro $\left.^{3}\right]$ )GIP is a stable and specific antagonist of the GIP receptor and has been shown to effectively antagonise the acute insulinotropic effect of GIP in vitro and in vivo, resulting in acute impairment of glucose ultimately culminates in type 2 diabetes. The benefits of compromised GIP action in obesity-related diabetes are envisaged to follow from diminished signalling at the adipocyte GIP-R. Any negative effect on beta cell GIP-Rs and insulin secretion in obesity-related diabetes is compensated by various factors including: (1) improved insulin sensitivity and diminished insulin demand; (2) alleviation of beta cell dysfunction; and (3) compensation by upregulation of the GLP-1 component of the enteroinsular axis. In addition, the insulinotropic action of GIP has been shown to be severely diminished in patients with untreated type 2 diabetes. For further discussion of these concepts, see text and Fig. 2

tolerance [7, 8]. In normal mice, sustained chemical knockout of GIP receptor signalling results in mild impairment of insulin secretion and glucose homeostasis [9]. However, the metabolic and hormonal disarray of obesity-related diabetes generates an entirely different scenario following long-term disruption of GIP signalling. Thus, as opposed to marginal impairment of glucose homeostasis, the effects of GIP receptor blockade on lipid metabolism and fat deposition significantly improve metabolic status in obesity-related insulin-resistant states. For example, daily administration of $\left(\mathrm{Pro}^{3}\right)$ GIP for just 11 days to $o b / o b$ mice resulted in significant reduction of hyperglycaemia plus $\mathrm{HbA}_{1 \mathrm{c}}$ and marked improvement of insulin 
resistance, glucose tolerance and both beta cell morphology and insulin secretion [10]. Furthermore, once-daily administration of the antagonist to young $o b / o b$ mice indicated that compromised GIP action could prevent the onset of hyperglycaemia and associated metabolic abnormalities prior to any effect on body weight [11].

Studies in wild-type mice with diet-induced obesity-related diabetes illustrate strikingly similar consequences of biological GIP deficiency induced by $\left(\operatorname{Pro}^{3}\right)$ GIP $[12,13]$. Thus, antagonism of GIP receptor by daily injection of $\left(\mathrm{Pro}^{3}\right) \mathrm{GIP}$ was able to essentially reverse or prevent obesity, together with many of the metabolic abnormalities associated with high-fat feeding, e.g. hyperglycaemia, impaired glucose tolerance and dyslipidaemia $[6,12,13]$. A key aspect of these observations was the rapid and significant restoration of insulin sensitivity and depletion of triacylglycerol deposits in liver and muscle. The latter represents a key pathogenic factor, given the well established link between development of insulin resistance and spill over of lipid accumulation at these sites [14]. Lowered glucagon levels could account for part of these observations, but no changes in serum glucagon levels were observed in high-fat fed mice or mice chronically treated with $\left(\mathrm{Pro}^{3}\right)$ GIP [11-13].

Further refinement of peptide chemistry may make it possible to increase the potency and duration of action of $\left(\right.$ Pro $\left.^{3}\right)$ GIP as a potential biological molecule to induce compromised GIP action. However, there is already little doubt that specific biological GIP receptor antagonists, based on N-terminal modifications of GIP, potentially represent an exciting new class of drugs to combat the obesity-related diabetes epidemic. Studies in human patients will be required to delineate the promise of GIP receptor antagonists. However, the value of therapeutic peptides is well illustrated by the use of insulin for the treatment of type 1 diabetes and their advantage of acting at specific receptors on defined target cells. The main disadvantage is the need for administration by injection.

\section{GIP receptor antagonism using low molecular weight molecules}

The potential of low molecular weight receptor ligands for oral administration has often been examined with a view to improving the therapeutic benefit of peptide-based pharmaceuticals and avoiding parenteral administration [15]. Although only reported in preliminary form to date, one study has assessed the metabolic effects of sustained downregulation of GIP signalling using a small molecule GIP receptor antagonist, SKL-14959. Administration of SKL-14959 to high-fat fed mice for 14 weeks significantly reduced body weight gain without affecting food intake [16]. Importantly, and in harmony with studies using $\left(\mathrm{Pro}^{3}\right)$
GIP, SKL-14959 administration was associated with depressed triacylglycerol accumulation in liver and muscle tissue in high-fat fed mice [16]. In a parallel study using diabetic $\mathrm{KK} A^{\mathrm{y}}$ mice, 4 weeks oral administration of SLK14959 significantly decreased plasma glucose and $\mathrm{HbA}_{1 \mathrm{c}}$ levels and ameliorated insulin resistance. These observations were also accompanied by depressed triacylglycerol accumulation in liver [16]. Thus, it appears that chemical GIP receptor blockade using $\left(\mathrm{Pro}^{3}\right)$ GIP or SLK-14959 is able to clear triacylglycerol deposits from liver of mice that lack GIP effect, thereby restoring mechanisms for suppression of hepatic glucose output. Comparatively very high doses of the small molecule antagonist are needed to induce these effects. Accordingly, further details of specificity, toxicity and chemical identity of SLK-14959 are needed to evaluate the potential therapeutic applicability of this approach, but these early results are encouraging.

\section{GIP vaccination}

Another approach to inhibition of GIP signalling by chemical means is the induction or administration of GIPspecific neutralising antibodies. Two independent 'proof of concept' studies have confirmed the therapeutic attractiveness of such approaches for obesity-related diabetes [17, 18]. In a study by Fulurija and colleagues, high titres of specific GIP antibodies were generated by covalent attachment of GIP peptides to virus-like particles followed by subcutaneous vaccination in high-fat fed mice. This resulted in production of GIP antibodies and protection against diet-induced obesity with significantly reduced body fat stores [17]. These observations are similar to those produced by specific chemical GIP receptor blockade in mice with diet-induced obesity $[12,13]$. Surprisingly, no benefit on glycaemic control was noted by Fulurija and colleagues, but active GIP immunisation was shown to produce a significant improvement of glucose tolerance and circulating glucose in a separate study in genetically obese $o b / o b$ mice [18]. Thus, vaccination against GIP resulting in antibody production appears to be a practical means of countering the metabolic abnormalities associated with dietor genetically induced obesity-related diabetes. However further preclinical safety and toxicology studies will be required before the therapeutic concept can be considered in humans.

\section{Inhibition or targeted destruction of GIP-secreting K-cells}

An alternative way to downregulate GIP receptor signalling is to inhibit hormone release from intestinal $\mathrm{K}$ cells. A 
potential advantage of this approach is that drugs may be able to target K-cells directly from the intestinal lumen, rather than using systemic delivery of GIP-based peptides or immunogens with the possibility of unwanted side effects at various sites. However, relatively little is known about K-cell signal transduction mechanisms; moreover, given the many similarities with glucagon-like peptide-1 (GLP-1) secretion from L-cells and insulin secretion from beta cells, this approach is unlikely to prove sufficiently specific. For example, although inhibition may be possible by antagonism of G-protein-coupled lipid receptors (GPCR) (i.e. GPCR119, GPCR120 or GPCR40) [19], the promiscuity of GIP receptors [20], plus stimulatory effects by other classes of nutrients on GIP secretion represent major obstacles. In addition, the K-cell has also been reported to produce xenin, a hormone that may be involved in glucose regulation and has recently been shown to possess potent inhibitory effects on feeding behaviour in rodents [21]. It is therefore questionable whether such theoretical approaches for GIP blockade are applicable. Notwithstanding this, specific K-cell destruction in transgenic mice with regulatory elements of the GIP promoter/ gene expressing an attenuated diphtheria toxin A was shown to protect against diet-induced obesity and ameliorate insulin resistance [22]. Thus, the basic observations with chemical or genetic GIP receptor blockade (see next section) are essentially replicated by targeted knockout of GIP-secreting K-cells in mice fed high-fat diet [22]. Although valuable to reinforce the concept of beneficial effects of compromised GIP action, it seems unlikely that the development of specific K-cell cytotoxic drugs will prove exploitable for therapeutic purposes.

\section{GIP receptor knockout mice}

Transgenic mice with knockout of the GIP receptor have been particularly useful in elucidating mechanisms that underlie beneficial effects of compromised GIP action on diet-induced obesity [23, 24]. GIP receptor knockout in mice fed normal diet had little effect, other than small impairment of glucose tolerance and insulin secretion [25]. However, genetic knockout of the GIP receptor in normal or $o b / o b$ mice fed a high-fat diet was shown to decrease respiratory quotient and increase oxygen consumption [23, 24]. This indicates that increased energy expenditure and preferential oxidation of fat as an energy source is key in the beneficial anti-obesity action seen in these animals $[23$, $24,26]$. This is generally in keeping with the well-known anabolic effects of GIP on lipid metabolism and fat deposition. Thus, inhibition of GIP receptor function in GIP receptor knockout mice fed a high-fat diet resulted in suppression of body weight gain, reduced adiposity, decreased tissue triacylglycerol stores, reduced insulin resistance and marked improvement of glucose tolerance $[23,24]$. These observations are very similar to those encountered with chemical GIP receptor blockade. Other indications of altered energy metabolism in GIP receptor knockout mice include increased uncoupling protein-1 and decreased beta-3 adrenergic receptor levels in brown and white adipose tissue, respectively [24]. These data suggest that GIP antagonism enhances energy expenditure in situations of excessive dietary fat intake.

In agreement with these initial observations, a recent study has shown that genetic GIP receptor knockout prevented ovariectomy-induced obesity in mice, extending the same basic observations to distinctly different metabolic settings [27]. It is clear from studies to date using chemical blockade or genetic knockout of GIP signalling that amelioration of insulin resistance is the key element underlying the improvement of metabolic status $[10,13$, 16, 23, 24]. Indeed, genetic compromise of GIP signalling has been shown to prevent the development of ageingassociated insulin resistance in mice through changes in body composition and energy expenditure [28]. This is further reinforced by observations that genetic disruption of GIP signalling increased adiponectin levels under conditions of high-fat feeding [29]. Thus, high-fat fed mice exhibited increased fat oxidation in peripheral tissues and decreased triacylglycerol deposition in liver and muscle, together with improved insulin sensitivity [26, 29].

\section{Lessons from animal models}

As indicated above, there is now a substantial body of evidence directly linking GIP to the efficient storage of ingested fat, thus conferring a pivotal role for GIP in the development of obesity due to chronic overnutrition with high-fat diets. This contrasts strongly with GLP-1, which does not interact with specific receptors on adipocytes, but exerts glucose-lowering effects through stimulation of insulin secretion and suppression of glucagon, gastric emptying and feeding [30]. Accordingly, inhibition of GIP receptor signalling appears to represent a novel therapeutic strategy for obesity-related diabetes, acting at various sites to improve insulin resistance and pancreatic beta cell function, and to promote depletion of adipose tissue mass (Fig. 1). Importantly, in concurrence with this concept, the complete absence of GIP signalling does not appear to result in any serious adverse effects. Despite the obvious negative effect on the insulinotropic GIP-mediated incretin effect, the beneficial effects of sustained GIP receptor blockade on lipid metabolism and fat deposition considerably offset any such impediments [6]. Moreover, clinical studies have shown that the insulinotropic effect of GIP is 
already compromised in obesity-related diabetes [31] and the possibility of upregulation of GLP-1 or neural arms of the enteroinsular axis in GIP receptor knockout mice has been indicated [32]. Improvement of insulin resistance is also linked to lower circulating insulin levels and beta cell rest $[10,11]$, thereby rendering the loss of any stimulatory effects of GIP on insulin synthesis and beta cell mass, as observed in cellular models [4, 33], less important.

Clearly, further studies are warranted to evaluate the clinical potential of GIP receptor antagonism for human obesity-related diabetes. To date there are no reports of the metabolic consequence of longer-term specific GIP receptor blockade in the clinical setting, although various pharmaceutical companies are involved with the above-mentioned preclinical studies. However, lessons may come from patients undergoing bypass surgery with surgical diversion of nutrient passage away from GIP-secreting K-cells in the upper small intestine $[6,34]$.

\section{Evidence from human bypass surgery}

Gastric bypass procedures are associated with dramatic early (1-4 weeks) post-operative improvements of glycaemic control and insulin sensitivity, importantly even before substantial weight loss [35-39]. Explanations of why bypass surgery, as opposed to restriction surgery (e.g. gastric band), achieves greater glucose-lowering efficacy and rapid amelioration of insulin resistance are very much sought after [40], with the precise mechanisms for the prominent and almost immediate restoration of glycaemic control and insulin sensitivity remaining unclear. Restrictive surgery reduces the amount of food passing through the whole small intestine, whereas bypass surgery directs all food to the jejunum $[34,35]$. Rapid delivery of nutrients to the distal small intestine and bowel will upregulate GLP-1 secretion and partly explain dramatic improvements in insulin secretion and pancreatic beta cell function both within weeks and long after surgery [36, 37]. Increase of GLP-1 and peptide YY, which is also secreted from L-cells, may additionally promote satiety and dietary compliance (Fig. 2). However, in our view elevated GLP-1 levels are unlikely to account for the rapid improvement of insulin sensitivity and the alleviation of diabetes observed in patients. Thus, patients with type 2 diabetes treated with twice daily injections of exendin-4 or liraglutide had upregulation of beta cell function and blood glucose control, but without major effects on insulin resistance [41, 42]. However, endogenously released GLP-1, as opposed to injected GLP-1 mimetics, may interact with afferent nerves at the site of release (small intestine) and project 'insulin sensitivity signals' through the central nervous system. Further, the possible role of other gastrointestinal peptide mediators such as ghrelin, obestatin and glucagon should not be overlooked. Indeed, the culmination of subtle changes in circulating levels of various regulatory hormones could be fundamental in the beneficial effects of bypass surgery.

It has been hypothesised that exclusion of nutrient transit from the upper small intestine results in inhibited secretion of a hormone promoting insulin resistance and deposition of fat stores [35]. Clearly, given the aforementioned array of beneficial effects following GIP receptor blockade in obesity-related diabetes, a reduction in circulating GIP levels by surgical exclusion of the K-cell abundant proximal small intestine could be a key factor in the success of these surgeries [40]. Consistent with this view, several recent studies have shown that GIP is decreased rapidly, in the fasting and most notably postprandial states, following Roux-en-Y and biliopancreatic diversion bypass surgery in humans [34-37, 43]. Interestingly, in some studies GIP levels were lowered significantly in diabetic, rather than non-diabetic obese patients [35, 36], highlighting the significant role of GIP-mediated actions in this metabolic scenario. In consequence, this kind of surgery can be viewed to reproduce many of the beneficial effects noted in animal models of compromised GIP action, including rapid improvement of insulin resistance and beta cell function (Fig. 2). Nonetheless, it is possible that other important effects are mediated through mechanisms other than GIP, including the surgical 'knockout' of an as yet undiscovered hormone from the upper gastrointestinal tract [37]. Interestingly, there appears to be no reduction of GIP levels in other studies, possibly reflecting subtle variations in technique, timing of sample collection, nutritional status and degree of surgical bypass [44, 45].

It is noteworthy that the rapid depletion of hepatic and myocytic triacylglycerol stores for improved metabolic control in bypass surgery patients has been highlighted elsewhere, but attributed to dietary restriction alone [14]. Although severe energy restriction is clearly an important contributor following these surgical procedures [46], this variable seems unlikely to account for the superior metabolic results of bypass as opposed to restrictive surgery. Nevertheless, it should be remembered that in many cases the gastric band is not adjusted until after several weeks, meaning that energy restriction may be less immediate after this procedure than after bypass surgery. Accordingly, the underlying principles outlined in the current review give credence to the idea that blockade of GIP receptor action offers promise as a new and potentially important approach to obesity-related diabetes. This could be particularly so in obese patients in whom surgery is contraindicated. Indeed given the expense, potential hazards and need for long-term patient management, 


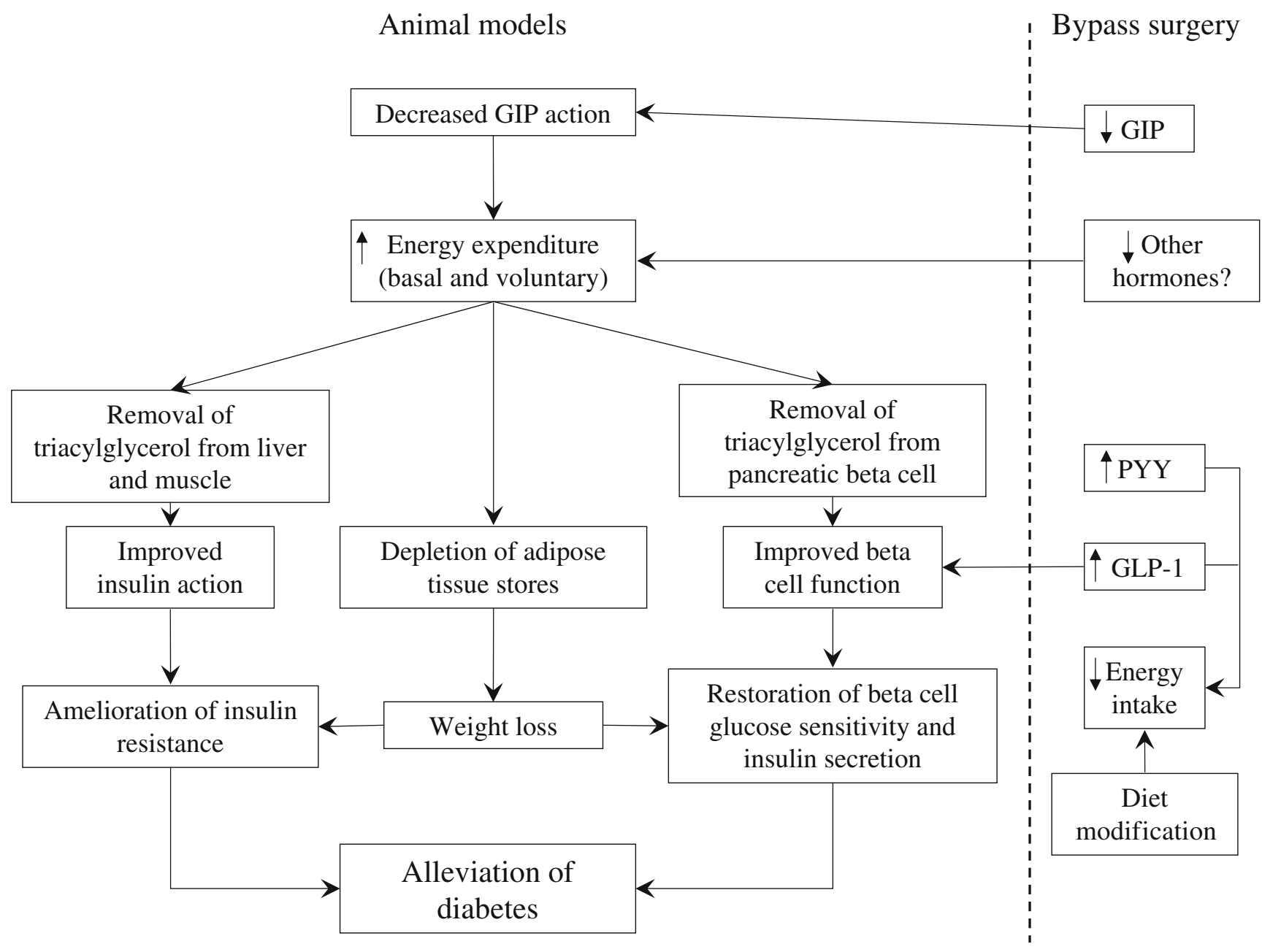

Fig. 2 Key events in mediating beneficial effects of compromised GIP action in animal models of obesity-related diabetes and parallels with clinical bypass surgery. In animals, increased energy expenditure resulting in removal of triacylglycerol from liver, muscle and pancreatic beta cells is regarded as key to the improvements in the action and secretion of insulin. Significant weight loss follows as the period of negative energy balance progresses. This is achieved without change in energy intake. In human bypass surgery, early benefits

alternatives to surgery are essential in the management of the obesity-related diabetes epidemic.

\section{Conclusion}

Growing evidence supports the view that compromise of GIP action serves to protect against development of obesity and associated metabolic disturbances in times of excessive caloric intake [6]. Thus, GIP appears to directly link overnutrition to obesity and is a potential novel target for anti-obesity-related diabetes drugs. The strikingly similar beneficial effect induced by a wide range of genetic and chemical approaches to impairment of GIP receptor signalling strengthens this belief. Interesting parallels also exist in include rapid amelioration of insulin resistance and improvement of beta cell function. The resulting alleviation of diabetes is associated with a notable decrease of circulating GIP and increases of GLP-1 and peptide YY (PYY). The latter hormones may contribute to body weight loss due to inhibition of energy intake. The possible involvement of other unknown hormone(s) and changes in energy expenditure after bypass surgery remain to be determined and merit investigation. Upward arrow, increased; downward arrow, decreased

clinically obese patients undergoing bypass surgery, with diversion of nutrient passage away from the gut section containing GIP-secreting cells. These patients display an unprecedented correction of hyperglycaemia and insulin sensitivity on a par with and possibly even greater than that observed in animal models with disruption of GIP receptor signalling. Further studies are needed to dissect the underlying mechanisms in these patients and to fully delineate the clinical potential of methods of GIP receptor blockade used to date and their possible application in man.

Acknowledgements The authors' work referred to in this review has been generously supported by Diabetes UK, University of Ulster strategic funding, and the SAAD Trading and Contracting Company (Al Khobar, Saudi Arabia). 
Duality of interest The authors hold stock in Diabetica Ltd, which has patents for exploitation of GIP receptor antagonists and other peptide therapeutics. P. R. Flatt also serves on scientific advisory boards and has received speaker's honoraria and research support from several companies with interests in glucose-lowering drugs and incretin-based therapies, including Astellas, MSD, Novartis, Novo Nordisk, Organon and Schering Plough.

\section{References}

1. Pederson RA, Schubert HE, Brown JC (1975) Gastric inhibitory polypeptide. Its physiologic release and insulinotropic action in the dog. Diabetes 24:1050-1056

2. Jörnvall H, Carlquist $M$, Kwauk $S$ et al (1981) Amino acid sequence and heterogeneity of gastric inhibitory polypeptide (GIP). FEBS Lett 123:205-210

3. Pederson RA, Schubert HE, Brown JC (1975) The insulinotropic action of gastric inhibitory polypeptide. Can J Physiol Pharmacol 53:217-223

4. Trümper A, Trümper K, Trusheim H, Arnold R, Göke B, Hörsch D (2001) Glucose-dependent insulinotropic polypeptide is a growth factor for beta (INS-1) cells by pleiotropic signaling. Mol Endocrinol 15:1559-1570

5. Yip RG, Boylan MO, Kieffer TJ, Wolfe MM (1998) Functional GIP receptors are present on adipocytes. Endocrinology 139:4004-4007

6. Flatt PR (2008) Dorothy Hodgkin Lecture, 2008. Gastric inhibitory polypeptide (GIP) revisited: a new therapeutic target for obesity-diabetes? Diabet Med 25:759-764

7. Gault VA, O'Harte FP, Harriott P, Flatt PR (2002) Characterization of the cellular and metabolic effects of a novel enzyme-resistant antagonist of glucose-dependent insulinotropic polypeptide. Biochem Biophys Res Commun 290:1420-1426

8. Gault VA, O'Harte FP, Harriott P, Mooney MH, Green BD, Flatt PR (2003) Effects of the novel (Pro3) GIP antagonist and exendin (9-39) amide on GIP- and GLP-1-induced cyclic AMP generation, insulin secretion and postprandial insulin release in obese diabetic (ob/ob) mice: evidence that GIP is the major physiological incretin. Diabetologia 46:222-230

9. Irwin N, Gault VA, Green BD et al (2004) Effects of short-term chemical ablation of the GIP receptor on insulin secretion, islet morphology and glucose homeostasis in mice. Biol Chem 385:845-852

10. Gault VA, Irwin N, Green BD et al (2005) Chemical ablation of gastric inhibitory polypeptide receptor action by daily (Pro3) GIP administration improves glucose tolerance and ameliorates insulin resistance and abnormalities of islet structure in obesity-related diabetes. Diabetes 54:2436-2446

11. Irwin N, McClean PL, O'Harte FP, Gault VA, Harriott P, Flatt PR (2007) Early administration of the glucose-dependent insulinotropic polypeptide receptor antagonist (Pro3) GIP prevents the development of diabetes and related metabolic abnormalities associated with genetically inherited obesity in $o b / o b$ mice. Diabetologia 50:1532-1540

12. McClean PL, Irwin N, Cassidy RS, Holst JJ, Gault VA, Flatt PR (2007) GIP receptor antagonism reverses obesity, insulin resistance, and associated metabolic disturbances induced in mice by prolonged consumption of high-fat diet. Am J Physiol Endocrinol Metab 293:E1746-E1755

13. Gault VA, McClean PL, Cassidy RS, Irwin N, Flatt PR (2007) Chemical gastric inhibitory polypeptide receptor antagonism protects against obesity, insulin resistance, glucose intolerance and associated disturbances in mice fed high-fat and cafeteria diets. Diabetologia 50:1752-1762
14. Taylor R (2008) Pathogenesis of type 2 diabetes: tracing the reverse route from cure to cause. Diabetologia 51:1781-1789

15. Cascieri MA, Koch GE, Ber E et al (1999) Characterization of a novel, non-peptidyl antagonist of the human glucagon receptor. J Biol Chem 274:8694-8697

16. Tsubamoto $\mathrm{Y}$, Nakamura $\mathrm{T}$, Kinoshita $\mathrm{H}$, Tanimoto $\mathrm{H}$, Noda $\mathrm{H}$ (2008) A novel low-molecular weight antagonist of glucosedependent insulinotropic polypeptide receptor, SKL14959, prevents obesity and insulin resistance. Diabetologia 51:S373 (Abstract)

17. Fulurija A, Lutz TA, Sladko K et al (2008) Vaccination against GIP for the treatment of obesity. PLoS ONE 3:e3163

18. Irwin N, McClean PL, Patterson S, Hunter K, Flatt PR (2009) Active immunisation against gastric inhibitory polypeptide (GIP) improves blood glucose control in an animal model of obesitydiabetes. Biol Chem 390:75-80

19. Parker HE, Habib AM, Rogers GJ, Gribble FM, Reimann F (2009) Nutrient-dependent secretion of glucose-dependent insulinotropic polypeptide from primary murine K cells. Diabetologia $52: 289-298$

20. Swaminath G (2008) Fatty acid binding receptors and their physiological role in type 2 diabetes. Arch Pharm (Weinheim) 341:753-761

21. Cooke JH, Patterson M, Patel SR et al (2009) Peripheral and central administration of xenin and neurotensin suppress food intake in rodents. Obesity (Silver Spring). doi:10.1038/oby2008.652

22. Althage MC, Ford EL, Wang S, Tso P, Polonsky KS, Wice BM (2008) Targeted ablation of glucose-dependent insulinotropic polypeptide-producing cells in transgenic mice reduces obesity and insulin resistance induced by a high fat diet. J Biol Chem 283:18365-18376

23. Miyawaki K, Yamada Y, Ban N et al (2002) Inhibition of gastric inhibitory polypeptide signaling prevents obesity. Nat Med 8: $738-742$

24. Hansotia T, Maida A, Flock G et al (2007) Extrapancreatic incretin receptors modulate glucose homeostasis, body weight, and energy expenditure. J Clin Invest 117:143-152

25. Miyawaki K, Yamada Y, Yano H et al (1999) Glucose intolerance caused by a defect in the entero-insular axis: a study in gastric inhibitory polypeptide receptor knockout mice. Proc Natl Acad Sci U S A 96:14843-14847

26. Zhou H, Yamada Y, Tsukiyama K et al (2005) Gastric inhibitory polypeptide modulates adiposity and fat oxidation under diminished insulin action. Biochem Biophys Res Commun 335:937-942

27. Isken F, Pfeiffer AF, Nogueiras R et al (2008) Deficiency of glucose-dependent insulinotropic polypeptide receptor prevents ovariectomy-induced obesity in mice. Am J Physiol Endocrinol Metab 295:E350-E355

28. Yamada C, Yamada Y, Tsukiyama K et al (2007) Genetic inactivation of GIP signaling reverses aging-associated insulin resistance through body composition changes. Biochem Biophys Res Commun 364:175-180

29. Naitoh R, Miyawaki K, Harada N et al (2008) Inhibition of GIP signaling modulates adiponectin levels under high-fat diet in mice. Biochem Biophys Res Commun 376:21-25

30. Baggio LL, Drucker DJ (2007) Biology of incretins: GLP-1 and GIP. Gastroenterology 132:2131-2157

31. Nauck MA, Heimesaat MM, Orskov C, Holst JJ, Ebert R, Creutzfeldt W (1993) Preserved incretin activity of glucagonlike peptide 1 [7-36 amide] but not of synthetic human gastric inhibitory polypeptide in patients with type-2 diabetes mellitus. J Clin Invest 91:301-307

32. Pamir N, Lynn FC, Buchan AM et al (2003) Glucose-dependent insulinotropic polypeptide receptor null mice exhibit compensatory changes in the enteroinsular axis. Am J Physiol Endocrinol Metab 284:E931-E939 
33. Gault VA, O'Harte FP, Flatt PR (2003) Glucose-dependent insulinotropic polypeptide (GIP): anti-diabetic and anti-obesity potential? Neuropeptides 37:253-263

34. Clements RH, Gonzalez QH, Long CI, Wittert G, Laws HL (2004) Hormonal changes after Roux-en Y gastric bypass for morbid obesity and the control of type-II diabetes mellitus. Am Surg 70:1-4

35. Rubino F, Gagner M, Gentileschi P et al (2004) The early effect of the Roux-en-Y gastric bypass on hormones involved in body weight regulation and glucose metabolism. Ann Surg 240:236242

36. Mingrone G, Nolfe G, Gissey GC et al (2009) Circadian rhythms of GIP and GLP1 in glucose-tolerant and in type 2 diabetic patients after biliopancreatic diversion. Diabetologia 52:873-881

37. Salinari S, Bertuzzi A, Asnaghi S, Guidone C, Manco M, Mingrone G (2009) First-phase insulin secretion restoration and differential response to glucose load depending on the route of administration in type 2 diabetic subjects after bariatric surgery. Diabetes Care 32:375-380

38. Mari A, Manco M, Guidone C et al (2006) Restoration of normal glucose tolerance in severely obese patients after bilio-pancreatic diversion: role of insulin sensitivity and beta cell function. Diabetologia 49:2136-2143

39. Guidone C, Manco M, Valera-Mora E et al (2006) Mechanisms of recovery from type 2 diabetes after malabsorptive bariatric surgery. Diabetes 55:2025-2031
40. Flatt PR (2007) Effective surgical treatment of obesity may be mediated by ablation of the lipogenic gut hormone gastric inhibitory polypeptide (GIP): evidence and clinical opportunity for development of new obesity-diabetes drugs? Diab Vasc Dis Res 4:151-153

41. Juhl CB, Hollingdal M, Sturis J et al (2002) Bedtime administration of NN2211, a long acting GLP-1 derivative, substantially reduces fasting and postprandial glycemia in type 2 diabetes. Diabetes 51:424-429

42. Egan JM, Meneilly GS, Elahi D (2003) Effects of 1-mo bolus subcutaneous administration of exendin-4 in type 2 diabetes. Am J Physiol Endocrinol Metab 284:E1072-E1079

43. Korner J, Bessler M, Inabnet W, Taveras C, Holst JJ (2007) Exaggerated glucagon-like peptide-1 and blunted glucosedependent insulinotropic peptide secretion are associated with Roux-en-Y gastric bypass but not adjustable gastric banding. Surg Obes Relat Dis 3:597-601

44. Whitson BA, Leslie DB, Kellogg TA et al (2007) Enteroendocrine changes after gastric bypass in diabetic and nondiabetic patients: a preliminary study. J Surg Res 141:31-39

45. Laferrère B, Heshka S, Wang K et al (2007) Incretin levels and effect are markedly enhanced 1 month after Roux-en-Y gastric bypass surgery in obese patients with type 2 diabetes. Diabetes Care 30:1709-1716

46. Greco AV, Mingrone G, Giancaterini A et al (2002) Insulin resistance in morbid obesity: reversal with intramyocellular fat depletion. Diabetes 51:144-151 